Guerra, D. S., Peñaloza, V. L., Quezado, I. \& Araújo, M. M. (2017). Análise das relações entre traços de personalidade, compra impulsiva e compra compulsiva. Consumer Behavior Review, 1(1) 24-37.

ISSN: 2526-7884

Editor: Prof. Dr. Marconi Freitas da Costa Email da revista: cbr@ufpe.br
Avaliação: Double blind review

Recebido: 18 de janeiro de 2017

Aceito: 19 de abril de 2017

\title{
ANÁLISE DAS RELAÇÕES ENTRE TRAÇOS DE PERSONALIDADE, COMPRA IMPULSIVA E COMPRA COMPULSIVA
}

\author{
Diego de Sousa Guerra \\ Verónica Lídia Peñaloza \\ Izabelle Quezado \\ Mirna Maia de Araújo
}

\begin{abstract}
Diego de Sousa Guerra é Professor Adjunto da Universidade Federal do Cariri. E-mail: diegoguerra@gmail.com. Verónica Peñaloza é Professora Adjunta da Universidade Estadual do Ceará. Email: vero.pf@hotmail.com. Izabelle Quezado é Doutoranda em Administração de Empresas pela Universidade de Fortaleza. E-mail: izabellequezado@gmail.com._Mirna Maia de Araújo é Professora da Faculdade Metropolitana da Grande Fortaleza. E-mail: mirnamaiaaraujo@gmail.com._Esta pesquisa teve o apoio da Fundação de Apoio ao Desenvolvimento Científico e Tecnológico do Ceará - FUNCAP. Os autores agracedecem aos avaliadores pelos comentários para melhoria do artigo.
\end{abstract}

\begin{abstract}
Resumo
Este estudo tem como objetivo identificar as relações existentes entre traços de personalidade, compra impulsiva (CI) e compra compulsiva (CC), bem como comparar os resultados com os achados de Sun, $\mathrm{Wu}$, Youn (2002). Para tanto, realizou-se uma pesquisa quantitativa por meio de survey com uma amostra de 424 participantes, dividida aleatoriamente em dois grupos de 212 participantes. Foram realizadas duas análises fatoriais confirmatórias em amostras diferentes e o modelo estrutural foi testado por meio dos softwares SPSS e AMOS. Os resultados indicam que neuroticismo e extroversão estão relacionados positivamente à $\mathrm{CI}$, bem como há uma associação positiva entre CI e CC. O trabalho apresenta uma contribuição metodológica sobre a forma de operacionalização das escalas e traz uma crítica à subespecificação da natureza da relação entre CI e CC. Palavras-chave: Traços de personalidade; Compra impulsiva; Compra compulsiva.
\end{abstract}

Esta obra está licenciado com uma Licença Creative Commons Atribuição 4.0 Internacional.

\section{INTRODUÇÃO}

Conhecer o consumidor e seu comportamento tem sido um dos principais desafios do marketing. Desde as primeiras décadas do século $\mathrm{XX}$, a visão economicista de homo economicus vem sendo substituída, gradativamente, pela introdução de estudos que avaliam a natureza comportamental no 
consumo (Verplanken \& Herabadi, 2001; Fontenelle, 2008).

A concepção de que indivíduos tomam decisões de compra pautadas exclusivamente em critérios racionais ou avaliações cognitivas das opções de mercado tem perdido espaço para a ideia de que as relações de compra são influenciadas pela ótica comportamental (Baker et al., 2016). Segundo Ferreira (2008), a explicação acerca do processo de consumo relacionado às variações entre oferta, demanda e o consumidor racional tem sido substituída pela influência dos pensamentos, das atitudes, das expectativas e dos sentimentos intrínsecos a esse processo.

Nesse sentido, as relações entre comportamento e personalidade são temas recorrentes na psicologia, desde Freud, e referências às relações entre personalidade e comportamento foram observadas, ainda, nos escritos ancestrais chineses, egípcios, na filosofia grega e europeia (Monteiro, Veiga \& Gonçalves, 2009). Para Baik et al. (2016), há indícios de que a personalidade é responsável por uma gama de variâncias no comportamento e nas intenções do indivíduo. Nessa ótica, variáveis de personalidade podem servir para diversos fins na área de marketing, indo desde o entendimento das preferências do consumidor até a previsão de comportamentos frente a determinados estímulos, como mídia e ações em ponto de venda (Shahjehan et al, 2016).

As respostas a esses estímulos geram sentimentos de urgência para a realização de uma compra, o que pode levar o indivíduo a deixar de realizar uma avaliação criteriosa das alternativas, adquirindo produtos e serviços sem considerar as consequências potenciais de sua opção, principalmente suas repercussões negativas (Costa, 2002). As consequências podem variar desde uma compra avaliada como inadequada (Rook, 1987) até a geração de comportamentos extremos (O'guinn \& Faber, 1989), principalmente a compulsão à compra e ao consumo, consideradas situações patológicas (Faber \& O'guinn, 1992). Essas situações podem ser caracterizadas como ações de CI e CC.

Em estudo desenvolvido no Brasil pelo Serviço de Proteção ao Crédito (SPC), identificou-se que $52 \%$ da amostra havia realizado uma compra por impulso nos três meses anteriores à pesquisa. Desse percentual, 41\% declarou estar endividado (Serviço de
Proteção ao Crédito, 2015). Ainda, a Associação Brasileira de Psiquiatria (ABP) alerta para a alta incidência de transtornos psicológicos associados ao consumo, com destaque à CC, identificada em 5\% da população (Associação Brasileira de Psiquiatria, 2012).

Frente a essa realidade, é ampla a investigação em torno da CI e da CC em pesquisas recentes em âmbito internacional (e.g. Tinne, 2011; Roberts \& Manolis, 2012; Lucas \& Koff, 2014; Baker et al., 2016; Baik et al., 2016) e nacional (e.g. Porpino \& Parente, 2013; Araújo \& Ramos, 2010; Dias et al., 2014).

Contudo, estudos que abordam a temática considerando traços de personalidade constituem-se de forma marginal nas pesquisas em Marketing (Sun, Wu \& Youn, 2002; Basso et al., 2009). Na maioria dos estudos, as relações encontradas foram fracas ou até inexistentes. Segundo Monteiro et al. (2009) as teorias sobre personalidade são, muitas vezes, conflitantes e os estudos da relação consumo e personalidade não revelaram uma boa capacidade preditiva no início, porém com a evolução das técnicas quantitativas, ressurge o interesse em verificar como os traços de personalidade podem ser relevantes na explicação do consumo.

Sun, Wu e Youn (2002) utilizaram um modelo de equações estruturais para avaliar as relações entre $\mathrm{CI}, \mathrm{CC}$ e traços de personalidade simultaneamente na mesma amostra. A comparação de seus resultados com a literatura da área é limitada, uma vez que os demais autores operacionalizam apenas duas dessas dimensões por estudo (e.g. Mowen \& Spears, 1999; Verplanken \& Herabadi, 2001).

Desta forma, este estudo tem como objetivo identificar as relações existentes entre traços de personalidade, $\mathrm{CI}$ e CC e comparar os resultados com os achados de Sun, Wu, Youn (2002). Para isso, utilizou-se uma amostra composta por estudantes universitários de uma região metropolitana do nordeste brasileiro. Assim, a questão principal para a qual se busca resposta por meio deste estudo é: qual a relação entre os comportamentos de CC, CI e os traços de personalidade?

Espera-se que esta investigação forneça dados psicométricos consistentes que subsidiem novos estudos envolvendo os construtos já enunciados, bem como apresente uma avaliação crítica das relações sob análise a partir de dados coletados no Brasil. Ademais, 
um aspecto que foi claramente negligenciado no modelo a ser avaliado é a explicação da relação entre CI e CC. Teoricamente, os dois construtos tem motivações diferentes e a explicação desta relação precisa ser melhor explorada. $\mathrm{Na}$ fase final do trabalho, buscou-se delimitar a natureza de cada um dos fenômenos, assim como procedeu-se uma análise crítica da relação de causa e efeito entre eles.

O artigo está estruturado em cinco seções, além desta introdução. 0 referencial teórico aborda a fundamentação conceitual dos fatores de personalidade e da CI e CC, com base nos principais paradigmas, bem como a formulação das hipóteses. Na sequência, são apresentados o método e as técnicas utilizadas na execução da pesquisa. Após, são discutidos os resultados à luz do referencial teórico. Nas considerações, por fim, são tecidas as principais exposições obtidas por meio da investigação empírica, bem como sugestões para pesquisas posteriores e as principais limitações.

\section{FUNDAMENTAÇÃO TEÓRICA}

Esta seção está divida em dois subitens. No primeiro, são dispostos os principais conceitos e abordagens do construto Traços de Personalidade. No segundo subitem, estão apresentadas as particularidades dos construtos CI e CC.

\section{Traços de personalidade}

A personalidade é um aspecto relevante na análise o comportamento do consumidor, uma vez que os tipos de personalidade podem ser classificados com precisão e que há fortes correlações entre certos tipos de personalidade e escolhas de produtos e marcas (Kassarjian, 1971; Baik et al., 2016). Para Pervin e John (2004), a personalidade representa as características do indivíduo que explicam padrões consistentes de sentimentos, pensamentos e comportamentos.

Ainda segundo Pervin \& John (2004), a personalidade pode ser entendida como um conjunto de padrões estáveis das dimensões afetivas, cognitivas e comportamentais do ser humano. Já para Basso (2008), a noção de personalidade pressupõe que cada indivíduo organiza suas próprias características de personalidade. Entretanto, ao se analisar diversos indivíduos, percebe-se que certos padrões surgem de características semelhantes. Assim, compreender aspectos inerentes à personalidade de um indivíduo pode ajudar a prever seu comportamento em uma determinada situação (e.g. Otero-López \& Villardefrancos, 2013).

Uma concepção central que permeia o estudo da personalidade é que as diferenças entre as personalidades individuais geram padrões de comportamentos diversos entre os indivíduos. Dessa forma, supõe-se que ao se conhecer a personalidade de um consumidor, seria possível prever e explicar suas tendências comportamentais (Baker et al., 2016).

$\mathrm{Na}$ área do marketing as três teorias que mais se adequam ao contexto do comportamento do consumidor são a teoria do controle, teoria dos traços e os princípios evolucionários da personalidade. Nos estudos contemporâneos, o modelo dos traços apresenta-se como a teoria de maior amplitude e influência, pois atua como um auxílio à forma como se interpreta o ambiente e se responde a ele, o que explica as diferenças individuais.

De acordo com Mowen (2000), esta teoria procura estudar as dimensões básicas da personalidade e tem se apresentado como promissora para essa área do conhecimento ao defender que a personalidade é composta por um conjunto de cinco fatores básicos e que a compreensão dos traços permite representar a individualidade em virtual plenitude (Davidoff, 1983). Esse enfoque auxilia na construção de métricas adequadas à utilização no marketing (Basso et al., 2009).

0 modelo dos cinco grandes fatores de personalidade sustenta que os traços com base biológica interagem com o ambiente social para orientar todos os comportamentos do indivíduo (Stock, Hippel \& Gillert, 2016). Saucier (1994) faz uso de quarenta adjetivos para mensurar os cinco fatores de personalidade, concluindo que a escala proposta por Goldberg (1992), composta por cem adjetivos, pode ser reduzida sem ter sua validade alterada.

Embora haja algumas controvérsias com relação à denominação de cada fator, Santos, Sisto e Martins (2003) adotam a seguinte tradução: extroversão, socialização, realização, neuroticismo e abertura. A extroversão, fator I, corresponde ao nível de sociabilidade de um indivíduo. Características como disposição, otimismo e afetuosidade fazem parte da 
definição de um indivíduo extrovertido (Silva et al., 2007). 0 fator II, socialização, refere-se aos tipos de interação e, de acordo com Santos, Sisto e Martins (2003), representam uma dimensão interpessoal. Silva et al. (2007) afirmam que este fator indica uma tendência a ser socialmente agradável, caloroso e dócil.

0 terceiro fator, realização, agrupa características de personalidade que revelam o grau de persistência, controle, organização e motivação para alcançar objetivos. As características do fator neuroticismo são associadas a dimensões neuróticas da personalidade, como ansiedade, depressão, tensão, irracionalidade, emoção. 0 último fator, abertura, refere-se a um comportamento exploratório e a busca de novas experiências, revelando que indivíduos que apresentam baixos níveis de abertura tendem a ser mais conservadores em suas preferências, além de apresentarem uma menor flexibilidade em suas crenças (Vasconcellos \& Hutz, 2008).

As relações propostas entre os cinco fatores de personalidade e a compra impulsiva, que correspondem ao primeiro nível de relação do modelo de Sun, Wu, Youn (2002), são apresentadas no Quadro 1 junto com os estudos que dão suporte a essas relações.

Quadro 1

Hipóteses e suporte teórico

\begin{tabular}{cccc} 
Hipótese & \multicolumn{1}{c}{ Relação } & Natureza & Estudos que suportam a relação \\
H1 & Neuroticismo-->CI & Positiva & $\begin{array}{c}\text { Sun, Wu \& Youn (2002); Shahjehan } \text { et al. (2012); Otero-López } \\
\text { \& Villardefrancos (2013); Shehzadi } \text { et al. (2016) }\end{array}$ \\
H2 & Socialização-->CI & Negativa & $\begin{array}{c}\text { Rook \& Hoch (1985); Piron (1991); Verplanken \& Herabadi } \\
\text { (2001) }\end{array}$ \\
H3 & Extroversão --> CI & Positiva & $\begin{array}{c}\text { Verplanken \& Herabadi (2001); Sun, Wu \& Youn (2002); } \\
\text { Shahjehan } \text { et al. (2012) }\end{array}$ \\
H4 & Abertura-->CI & Positiva & $\begin{array}{c}\text { Sun, Wu \& Youn (2002); Shahjehan } \text { et al. (2012); Shehzadi } \text { et } \\
\text { al. (2016) }\end{array}$ \\
H5 & Realização-->CI & Positiva & $\begin{array}{c}\text { Verplanken \& Herabadi (2001); Shahjehan } \text { et al. (2012); } \\
\text { Otero-López \& Villardefrancos (2013) }\end{array}$
\end{tabular}

Fonte: Elaborado pelos autores

Para Mowen (2000), esta abordagem é a mais indicada para teste de modelo e teorias de forma objetiva e independente, formando generalizações que suportem a criação de conhecimento aplicável a diversos campos do conhecimento, dentre eles o marketing. 0 modelo é apresentado na Figura 1.

Uma ressalva importante a ser feita acerca da relação negativa de socialização e CI, suportada por Verplanken e Herabadi (2001), Rook e Hoch (1985) e Piron (1991), é que em estudo recente, Shehzadi et al. (2016) encontraram uma relação de natureza inversa, ou seja, positiva. Entretanto, optou-se pela manutenção da hipótese com uma relação negativa, uma vez que há amplo suporte na literatura para essa decisão.

\section{Compra impulsiva (CI) e compra compulsiva (CC)}

Durante o Século XX predominou o modelo que defendia que os consumidores avaliam racionalmente as melhores opções disponíveis para a tomada de qualquer decisão de compra. Entretanto, segundo Aravena et al. (2006), vários pesquisadores têm mostrado que a maioria dos consumidores faz suas compras com base em intuições e impulsos.

Segundo Costa (2002), os impulsos experimentados pelo consumidor o conduzem a agir sem planejamento, não havendo uma avaliação cognitiva elaborada sobre a adequação da compra naquele momento. Para Lucas e Koff (2014), essa impulsividade pode ser vista como algo disfuncional, quando o 
sujeito não pensa, não reflete, sendo este ato involuntário e incontrolável.

Nos indivíduos em que o estado afetivo supera a cognição, o comportamento impulsivo de compra se torna mais provável. 0 senso de espontaneidade e imediatismo dos impulsos de compra leva a um mínimo esforço de processamento de informações, com um reduzido esforço de deliberação cognitiva (Youn, 2000), podendo resultar em comportamentos indesejáveis.

\section{Figura 1}

Modelo de Sun, Wu e Youn (2002)

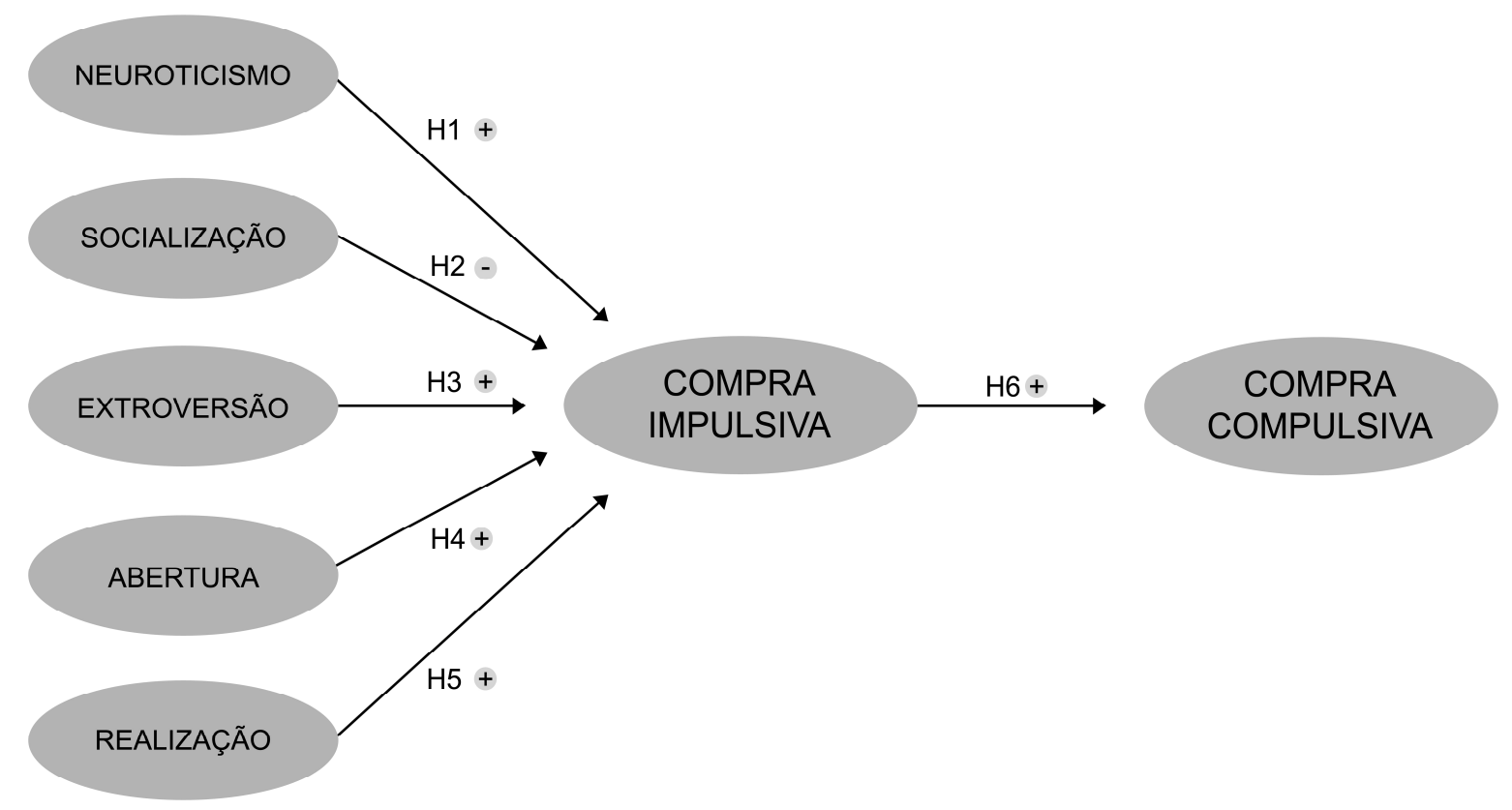

Nota. Fonte: Elaborada pelos autores com base em Sun, Wu e Youn (2002)

A inclinação em distorcer o valor das consequências do ato impulsivo, subestimandoas, conduz o indivíduo a render-se à tentação e agir impulsivamente. A ênfase do momento presente, representada pela desconsideração das consequências e repercussões no futuro, é um componente cognitivo fundamental da CI e da CC (Rook, 1987; Beatty \& Ferrel, 1998; Youn, 2000).

É importante diferenciar CI, que é uma resposta momentânea e pontual motivada pela mídia e outros elementos do mix de marketing; da CC, que é recorrente, irracional e motivada internamente por sentimentos e emoções negativas (Pinto, 2003).

A CC é uma forma de compulsão, exacerbada em um padrão de consumo anormal, no qual indivíduos se entregam a ímpetos incontroláveis de compra (Faber \& O'Guinn, 1992). Como assinalam Matos e Bonfanti (2008), o comportamento compulsivo geralmente é influenciado por motivadores internos, estados emocionais negativos, como tensão, ansiedade e tristeza; e ajuda a fornecer um alívio para esses sentimentos.

Já a CI ocorre quando um consumidor experimenta uma necessidade repentina, persistente e muitas vezes poderosa de comprar algo imediatamente. Esse impulso de compra é hedonicamente complexo e pode estimular conflitos de natureza emocional. Além disso, ocorre com reduzida preocupação com suas consequências (Rook, 1987).

Para Aravena et al. (2006), o lado patológico da compra por impulso pode ser caracterizado pela $\mathrm{CC}$, que surge como um extremo daquele comportamento. Dessa forma, quando a ausência de controle dos impulsos é acumulada até um ponto em que ocorre a perda crônica de controle do impulso, a CI pode evoluir para a CC (Zanella, 2001). 
De acordo com o discurso de Monteiro (2006), alguns autores têm proposto que características da personalidade estão intimamente relacionadas ao consumo compulsivo (e,g, O'Guinn \& Faber, 1989; Mowen, 2000). Piron (1991), por exemplo, propôs que todas as compras são acompanhadas de fatores emocionais e/ou reações cognitivas. Para Mowen e Spears (1999), os comportamentos de CC e CI parecem estar relacionados a uma perda de controle. Sun, Wu e Youn, (2002) sugerem que tanto a CI quanto a CC estão relacionadas aos mesmos traços psicológicos.

No estudo de Sun, Wu e Youn (2002) os autores desenvolveram um modelo de equações estruturais para investigar as relações entre fatores de personalidade, a $\mathrm{CI}$ e a CC. Como resultado, foram encontradas relações estatisticamente significativas entre o neuroticismo, a extroversão e a abertura com a CI. A relação positiva entre CI e CC também apresentou relevância estatística.

Outro estudo realizado por Mowen (2000) correlacionou a impulsividade (não a CI) aos traços de personalidade, ao materialismo, à excitação e à CC. Os resultados revelaram que o neuroticismo, a extroversão e a realização foram negativamente relacionadas a uma escala de impulsividade. 0 materialismo e abertura foram positivamente relacionados com a impulsividade, mesma relação foi observada entre impulsividade e CC.

Esses resultados revelam que o comportamento impulsivo de compra tende a ser motivado por gratificação imediata, e menos provável pelos traços de personalidade interiores. Por outro lado, o estudo demonstrou que uma quantidade significativa de variância da CC pode ser explicada pelo neuroticismo e, principalmente, a $\mathrm{CI}$, o que fundamenta o argumento de que as compras compulsivas são ativadas pela tensão psicológica (Valence et al., 2004) e respondem a sentimentos negativos ou eventos (Faber \& O'guinn, 1992).

Com base nos estudos de Aravena et al. (2006), Mowen (2000) e Sun, Wu e Youn (2002), sugere-se H6: A compra impulsiva (CI) influencia positivamente a compra compulsiva (CC).

\section{MÉTODO}

Trata-se de uma pesquisa descritiva, direta, com dados de corte transversal, utilização do método de survey e coleta estruturada de dados. Participaram da pesquisa 424 estudantes universitários de uma região metropolitana do nordeste, divididos aleatoriamente em duas amostras iguais de 212 indivíduos. A amostra utilizada é de natureza não-probabilística, por conveniência.

0 procedimento para a coleta de dados teve como base um questionário dividido em quatro seções. A primeira avalia o comportamento de CI por meio da utilização da escala de Rook e Fisher (1995). Esta escala, composta por nove assertivas, considera a questão cognitiva da impulsividade de compra a partir do reconhecimento que o indivíduo pode avaliar nas repercussões sociais do seu ato impulsivo (Costa, 2002).

A segunda seção determina o comportamento de CC através da escala Diagnostic Screener for Compulsive Buying (DSCB) desenvolvida por Faber e O'Guinn (1992), com o intuito de identificar quais dos entrevistados apresentavam atitudes compatíveis com compradores compulsivos.

A seção seguinte utiliza a escala composta por quarenta adjetivos proposta por Saucier (1994) para examinar os cinco fatores de personalidade (5FP) dos respondentes. A última seção traça o perfil sociodemográfico da amostra.

As escalas utilizadas neste trabalho sofreram adaptações das originais, uma vez que os trabalhos de Rook e Fisher (1995) e Faber e O'Guinn (1992) utilizaram um likert de 5 pontos, a primeira variando de "discordo totalmente" à "concordo totalmente" e a segunda de "nunca" a "muito frequentemente". Para este trabalho, utilizou-se uma escala intervalar, variando de 0 para "discordo totalmente" até 10 para "concordo totalmente" para as seções um e dois, e, para a seção 3, variando de 0 para "extrema imprecisão" até 10 para "extrema precisão".

Esta adaptação foi realizada por se acreditar que a familiaridade que as pessoas têm no Brasil de lidar com o sistema decimal em situações cotidianas de avaliação pode minimizar erros de mensuração da escala, especialmente em estratos de elevado nível de instrução. Espera-se que um maior número de opções melhore a precisão da escala sem 
aumentar os erros de mensuração, "permitindo ao instrumento diferenciar objetos (indivíduos) e atributos (traços), ao mesmo tempo em que identifica correlações mais próximas da realidade" (Monteiro, 2006, p.82).

Foi aplicado um pré-teste para identificar possíveis falhas e minimizar problemas na aplicação do instrumento de pesquisa. Um recorte na amostragem selecionou 15 respondentes. Esta é a quantidade mínima exigida para um pré-teste consistente (Almeida \& Botelho, 2006). Após o processo de tradução reversa, percebeu-se a necessidade de adaptações nas traduções dos adjetivos utilizados na escala de personalidade de Saucier (1994). Após esta adequação, a incongruência foi solucionada de maneira que não alterasse a escala validada.

Os missing values, isto é, as não-respostas a determinado item do questionário foram tratadas utilizando o método de substituição pela média dos dados presentes daquela variável. Os índices de não-resposta variaram de $0,23 \%$ a $0,94 \%$, estando, portanto, dentro da faixa recomendada pela literatura especializada, que é de até 10\% (Costa, 2002). Outro pressuposto testado foi a ausência de outliers, ou seja, padrões de resposta destoantes das demais observações da amostra. Quinze questionários que apresentaram escores $\mathrm{Z}$ maiores que $|3|$ em algumas das variáveis foram excluídos da amostra (Costa, 2002).

A análise da validade e confiabilidade das escalas foi realizada em duas etapas, com amostras distintas. Na etapa inicial, utilizando a primeira amostra, foi executada uma análise fatorial confirmatória (AFC), com o propósito de avaliar a possibilidade de replicação do modelo teórico aos dados da pesquisa (Worthington \& Whitaker, 2006). Na segunda etapa, utilizando a segunda amostra, os dados foram submetidos a uma nova $\mathrm{AFC}$, visando ratificar a estrutura fatorial obtida na primeira análise.

Neste estudo, optou-se pela realização de dois procedimentos de $\mathrm{AFC}$ em razão da natureza da pesquisa. É comum na literatura de marketing que análises como as procedidas neste manuscrito sejam conduzidas com uma análise fatorial exploratória (AFE) prévia à $\mathrm{AFC}$, entretanto, autores como Montavani e Bido (2015) e Worthington e Whittaker (2006) sugerem a adequação deste procedimento em trabalhos que se propõem a desenvolver instrumentos de mensuração ou verificar a unidimensionalidade de construstos Conway e Huffcut (2003).

Esta posição é reforçada pela distinção entre as técnicas proposta por Joreskog (2007) , em que a AFE é adequada para a definição da quantidade de fatores, a explicação de suas intercorrelações e a definição de quais fatores estão sendo mensurados, enquanto que na AFC, os fatores são conhecidos previamente conhecidos e tem sua estrutura fatorial fortemente amparado pela teoria do campo, como é o caso deste estudo.

Finalmente, o último procedimento de tratamento dos dados foi o teste do modelo estrutural, a partir de modelagem de equações estruturais (MEE), utilizando-se o software AMOS 18.0. Todos os procedimentos desta etapa foram operacionalizados através do método de maximum likelihood estimation (MLE).

\section{RESULTADOS}

Dispõe-se, neste tópico, os resultados da pesquisa empírica, por meio de caracterização da amostra, análise fatorial confirmatória, avaliação e teste do modelo e, por fim, apresenta-se uma discussão em torno de tais resultados.

\section{Caracterização das amostras e análise fatorial confirmatória}

A primeira amostra é composta por 126 homens $(59,4 \%)$ e 86 mulheres $(40,6 \%)$. A maioria dos respondentes é solteira $(73,6 \%)$, sem filhos $(76,4 \%)$, idade entre 20 e 25 anos $(59,1 \%)$, mora com os pais $(59,9 \%)$ e trabalha $(59,9 \%)$.

O primeiro grupo foi submetido a uma análise confirmatória prévia com o objetivo de remover os itens cujos erros têm elevada covariância com erros de outros itens, em um mesmo construto. A solução para esse problema é a eliminação de indicadores problemáticos por meio dos índices de modificação fornecidos pelo software de análise. Busca-se, dessa maneira, a purificação de cada escala utilizada e consequentemente um maior ajustamento do modelo de mensuração.

Os índices de ajustamento considerados para essa etapa foram: GFI, TLI e CFI superiores a 0,90, RMSEA inferior a 0,08, CMIN/DF $<5$. 
Adicionalmente, foi calculado o Alpha de Cronbach com o propósito de se avaliar a confiabilidade do construto. Os valores de Alpha considerados como adequados foram maiores que 0,600, conforme Hair Jr. Et al. (2009). Os resultados da primeira AFC são apresentados e comentados a seguir, na Tabela 1.

Tabela 1

Resultados da primeira AFC para a escala de 5FP

\begin{tabular}{lccccc}
\hline & Neuroticismo & Socialização & Extroversão & Abertura & Realização \\
\hline CMIN/DF & 1,236 & 1,223 & 1,046 & 27,046 & 26,013 \\
GFI & 0,996 & 0,994 & 0,997 & 0,926 & 0,928 \\
CFI & 0,997 & 0,996 & 1,000 & 0,671 & 0,818 \\
TLI & 0,992 & 0,988 & 0,999 & 0,012 & 0,455 \\
RMSEA & 0,033 & 0,032 & 0,015 & 0,351 & 0,344 \\
Alfa & 0,642 & 0,651 & 0,667 & - & - \\
\hline
\end{tabular}

Fonte: Elaborada pelos autores.

Três dos cinco construtos de traço de personalidade (neuroticismo, socialização e extroversão) apresentaram índices de ajuste satisfatórios, uma vez que todos os critérios de avaliação estão de acordo com os padrões adotados. A obtenção desse ajuste foi possível após testes, que resultaram na exclusão do itens não invejoso, relaxado melancólico, ciumento e invejoso, para o neuroticismo; audacioso, enérgico, tímido, quieto e inquieto para extroversão; e simpático, amável, caloroso e cooperativo para o construto socialização.
Os demais construtos foram excluídos da análise por apresentarem índices muito aquém dos estabelecidos como adequados para este tipo de análise. As dimensões ajustadas foram submetidas a uma nova AFC com uma amostra distinta da utilizada no primeiro teste e os índices obtidos são apresentados na tabela 2 .

A segunda amostra é composta por 135 homens $(63,7 \%)$ e 77 mulheres (36,3\%). A maioria dos respondentes é solteira (92\%), sem filhos (92,5\%), idade entre 20 e 25 anos $(64,6 \%)$, mora com os pais $(74,5 \%)$ e trabalha $(55,7 \%)$.

Tabela 2

Resultados da segunda AFC para a escala de 5FP

\begin{tabular}{lccc}
\hline & Neuroticismo & Socialização & Extroversão \\
\hline CMIN/DF & 4,734 & 2,748 & 0,648 \\
GFI & 0,985 & 0,987 & 0,998 \\
CFI & 0,942 & 0,969 & 1,000 \\
TLI & 0,827 & 0,907 & 1,000 \\
RMSEA & 0,063 & 0,047 & 0,000 \\
Alfa & 0,620 & 0,651 & 0,672 \\
\hline
\end{tabular}

Fonte: Elaborada pelos autores.

Nesta etapa, os ajustes foram obtidos sem a exclusão de itens adicionais, uma vez que que as alterações propostas não apresentavam ganhos de ajuste que justificasse a exclusão de qualquer dos itens. Ao final do processo, a referida escala passou a ser representada no modelo pelas dimensões neuroticismo (temperamental, obsessivo e irritável), socialização (frio, antipático, rude e severo) e extroversão (comunicativo, extrovertido e acanhado).

0 mesmo procedimento confirmatório foi adotado para a escalas de CC e CI. No primeiro teste confirmatório, utilizando a primeira amostra, os dados foram submetidos a seis rodadas de avaliação, resultando na exclusão de seis itens da escala de CI e dois itens da escala de CC. Com essas exclusões, foi possível alcançar 
os itens de ajustamento enunciados no início desta seção, conforme pode ser observado na
Tabela 3.

Tabela 3

Resultados da AFC para as duas amostras de $\mathrm{Cl}$ e CC

\begin{tabular}{lcccc}
\hline & CI (amostra 1) & CI (amostra 2) & CC (amostra 1) & CC (amostra 2) \\
\hline CMIN/DF & 1,613 & 2,176 & 1,361 & 1,062 \\
GFI & 0,963 & 0,953 & 0,988 & 0,990 \\
CFI & 0,983 & 0,945 & 0,992 & 0,995 \\
TLI & 0,976 & 0,923 & 0,984 & 0,991 \\
RMSEA & 0,054 & 0,075 & 0,041 & 0,017 \\
Alfa & 0,887 & 0,772 & 0,726 & 0,765 \\
\hline
\end{tabular}

Fonte: Elaborada pelos autores.

Após as adequações guiadas pelos modification índices, os dados foram novamente submetidos a uma AFC, agora com uma segunda amostra, como o propósito de verificar a possibilidade de reaplicação do modelo a uma amostra diferente da utilizada para o seu ajuste. Esta possibilidade foi confirmada e não houve a necessidade de exclusões adicionais de ajuste. Os índices finais de ajustamento das duas dimensões podem ser observados nas colunas que contemplam a segunda amostra na Tabela 3.

\section{Avaliação e teste do modelo}

$\mathrm{O}$ modelo proposto por Sun, $\mathrm{Wu}$, Youn (2002), mostrado na Figura 1, utiliza cinco construtos para mensurar traços de personalidade, um para a CI e um para a CC. Entretanto, os resultados empíricos de não ajustamento dos construtos realização e abertura, ainda na fase confirmatória do estudo, e sua consequente eliminação, sugerem a necessidade de adequação do modelo proposto, conforme apresentado na Figura 2.

Visando a mitigar interações indesejáveis entre os diversos construtos utilizados na pesquisa, avaliou-se a possibilidade da eliminação de indicadores com alta covariância entre si, entretanto, todas as recomendações de eliminação sugeridas pelos modification índices geraram ganhos marginais de ajuste que não sustentavam a exclusão desses indicadores, mantendo-se, portanto, as estruturas obtidas com as AFC.

0 modelo de mensuração final apresentou um ajuste marginal, com CMIN/DF $=1,644$, RMSEA $=0,055, \mathrm{GFI}=0,853, \mathrm{CFI}=0,849$ e $\mathrm{TLI}=$ 0,827 . Três dos cinco indicadores (GFI, CLI e TLI) apresentaram ajustes marginais, que foram considerados satisfatórios dada a natureza exploratória do trabalho.

Figura 2

Modelo adaptado

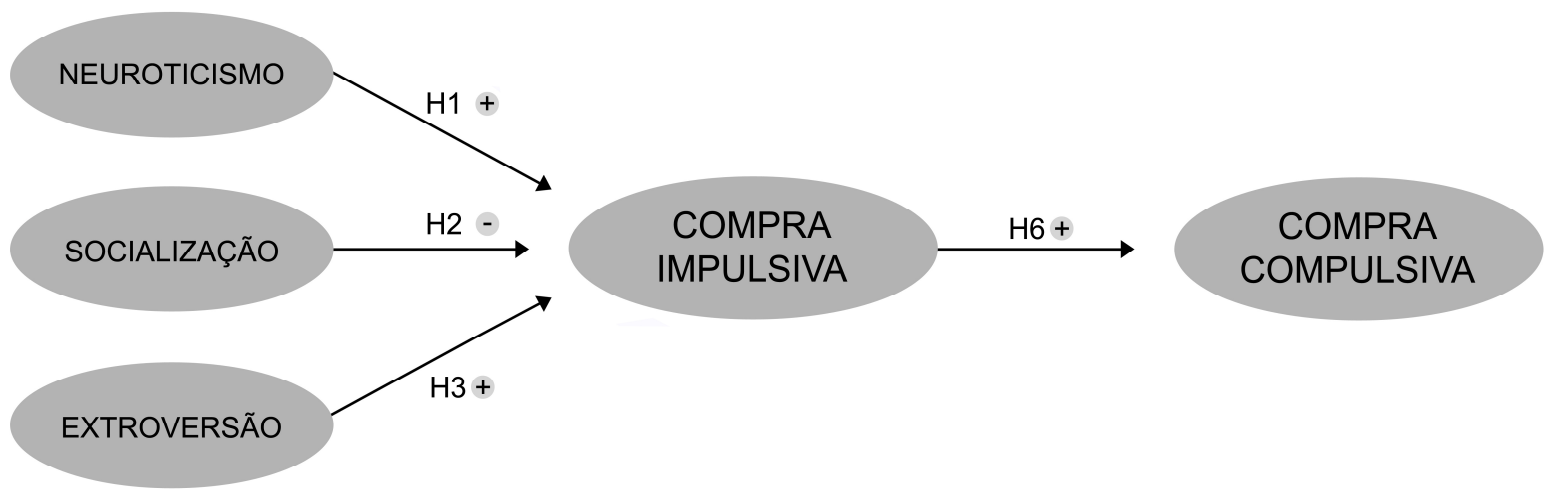

Fonte: Elaborada pelos autores. 
Para o teste de hipótese, foram adotados como significantes os resultados que apresentarem $\rho<0,10$ (Sun, Wu \& Youn, 2002). Considerou-se tal índice a fim de manter o parâmetro de comparação com o estudo proposto originalmente. Os resultados dos testes de hipótese podem ser observados a seguir, na Tabela 4.

Tabela 4

Resultados do modelo

\begin{tabular}{lccccc}
\hline \multicolumn{1}{c}{ Hipótese } & Estimate & S.E & C.R & SIG & Status \\
\hline Neuroticismo --> CI & 0,471 & 0,146 & 3,221 & 0,001 & Aceita \\
Socialização --> CI & 0,222 & 0,162 & 1,370 & 0,171 & Rejeitada \\
Extroversão --> CI & 0,488 & 0,210 & 2,328 & 0,020 & Aceita \\
CI --> CC & 0,658 & 0,078 & 8,393 & 0,000 & Aceita \\
\hline
\end{tabular}

Fonte: Elaborada pelos autores .

Observa-se que há evidências estatísticas suficientes para relacionar positivamente o neuroticismo à $\mathrm{CI}$, fazendo com que $\mathbf{H 1}$ seja aceita (valor-p=0,001). Estes resultados convergem com os resultados oferecidos Shahjehan et al. (2012), Shehzadi et al. (2016) e Otero-López \& Villardefrancos (2013), bem como com os achados de Sun, Wu e Youn (2002), entretanto, neste manuscrito, a quantidade de variação em CI acrescida pela acréscimo de uma unidade do neuroticismo é quase o triplo da observada no estudo original, o que sugere que a relação pode ter sido subestimada anteriormente.

A relação negativa entre a socialização e a CI não apresentou significância estatística (Estimate $=0,222$; valor-p $=0,171$ ), fazendo com que $\mathbf{H 2}$ seja refutada. Os resultados mostraram que, apesar de não significativa, a relação entre os construtos tem natureza positiva, contrariando Rook e Hoch (1985), Piron (1991) e Verplanken e Herabadi (2001) e corroborando os achados mais recentes de Shehzadi et al. (2016). Uma explicação teórica plausível para esta relação parece emergir do nível de interação dos indivíduos mais sociáveis com os ambientes de compra, bem como do contato com vendedores, que os expõem mais diretamente aos esforços de marketing depreendidos pelos ofertantes e, consequentemente, os levam a uma maior incidência de compra impulsiva (Donovan et al., 1994).

A extroversão foi confirmada como antecedente da CI, tornando H3 aceitável (valor-p=0,020). Esta hipótese supunha um relacionamento positivo entre as duas dimensões. Tais resultados fornecem evidências que ratificam as descobertas do estudo original e dos trabalhos de Verplanken \& Herabadi (2001) e Shahjehan et al. (2012).

$\mathrm{Na}$ Tabela 4, pode-se observar que a extroversão é responsável por aproximadamente $48 \%$ da variação da CI, mantendo-se todas as outras variáveis constantes, o que, de forma bastante consistente, sustenta a proposição teórica do relacionamento positivo entre os dois constructos.

De maneira análoga ao neuroticismo, a variação de CI explicada pela extroversão foi três vezes maior neste estudo do que no estudo original conduzido nos Estados Unidos. Sun, $\mathrm{Wu}$ e Youn (2002) utilizaram uma escala de 7 pontos, que variava de -3 a 3 , enquanto neste estudo foi utilizada uma escala de 11 pontos. Essa diferença permite a captação uma variabilidade, aproximadamente, $57 \%$ maior no segundo caso em relação ao primeiro. Desta forma, aumenta-se a precisão da escala e, consequentemente, evidencia-se melhor as relações propostas pela rede nomológica.

$\mathrm{O}$ relacionamento positivo entre $\mathrm{CI}$ e CC foi verificado (valor-p=0,000), corroborando H6. A confirmação desta hipótese reforça a literatura subjacente ao modelo teórico, que propõe que CI é antecedente de CC. Dessa forma, quando há uma perda crônica de controle do impulso, CI pode evoluir para CC (Zanella, 2001), o que corrobora a ideia de que CI antecede CC, defendido por Aravena et al. (2006).

Neste ponto, apesar de haver fortes indícios da relação entre os construtos, há a necessidade de maior aprofundamento dos estudos para 
identificar como há a transição entre uma resposta positiva a estímulos externos do $m i x$ marketing (CI) e o comportamento repetitivo de descontrole sistemático do impulso (CC), que é caracterizado por respostas a sentimentos negativos, como estresse e ansiedade (Roberts, Manolis \& Pulling, 2014; Kukar-Kinney, Scheinbaum \& Schaefers 2016).

Finalmente, não foi possível testar as hipóteses H4 e H5, uma vez que os construtos antecedentes associados a estas hipóteses foram excluídos no processo de AFC.

\section{CONCLUSÃO}

0 estudo das relações entre $\mathrm{CI}, \mathrm{CC}$ e traços de personalidade, apesar de bastante relevante, não tem recebido a devida atenção por parte da academia como foco de investigação, isto é evidenciado pela quantidade modesta de estudos que tratam destas questões, tanto no Brasil quanto no exterior.

Algumas pesquisas vêm sendo desenvolvidas relacionando apenas dois desses construtos (e.g. Lucas \& Koff, 2014), sem aprofundar as relações entre as três dimensões na mesma amostra. Nessa perspectiva, a pesquisa aqui desenvolvida possibilitou a construção de uma compreensão atualizada da relação entre os cinco grandes fatores de personalidade de Saucier (2004), CC e CI por apresentar um modelo integrativo no contexto brasileiro, alcançando, assim, o objetivo proposto de identificar as relações existentes entre traços de personalidade $\mathrm{CI}$ e CC, bem como comparar os resultados com os achados de Sun, $\mathrm{Wu}$, Youn (2002).

Em relação a essa comparação de resultados, há dois pontos mais relevantes. No trabalho original, de Sun, Wu e Youn (2002), o construto abertura mostrou-se um antecedente de CI, entretanto, esta hipótese não pôde ser testada no presente trabalho em razão da exclusão desta dimensão ainda na fase confirmatória. Já a dimensão socialização e sua relação com $\mathrm{CI}$, que não foi significativa nos dois trabalhos, apresentou relações conflitantes, sendo negativa no primeiro e positiva no segundo manuscrito.

Como já discutido na seção anterior, os resultados aqui apresentados de uma relação positiva com a CI parecem ser mais consistentes teoricamente, uma vez que o aumento da socialização geraria uma maior exposição do indivíduo aos estímulos ambientais e ao poder de persuasão dos vendedores, gerando, consequentemente, um maior índice de compras por impulso.

Este estudo também trouxe uma contribuição metodológica relevante relacionada à forma de mensuração das dimensões quando comparado ao trabalho original de Sun, Wu e Youn (2002) por utilizar uma escala de 11 pontos ao invés de sete. Desta forma, ganhou-se e consistência psicométrica, apesar de haver uma mitigação na segurança das respostas. 0 resultado desse ganho psicométrico está associado, provavelmente, à uma evidenciação mais intensa das relações entre neuroticismo/extroversão e CI.

Ainda em termos metodológicos, há a constatação da exclusão de duas dimensões da escala de traços de personalidade, ainda na fase confirmatória do estudo, que sucinta uma crítica ao instrumento de Saucier (1994). 0 uso de adjetivos pouco usuais no vocabulário do brasileiro médio, como "absorto", ou a utilização de itens semanticamente iguais, mas como valências trocadas como itens do mesmo construto, como "criativo" e "não criativo", parecem limitar a mensuração dos traços de personalidade e, consequentemente, da suas relações com outros construtos.

Os resultados deste estudo indicam, adicionalmente, a importância de novas investigações sobre as relações existentes entre a CI e a CC, corroborando as indicações teóricas sobre a relevância deste tipo de estudo e da amplitude de possibilidades para novas pesquisas. A condução de estudos qualitativos envolvendo CI e CC pode ser bastante útil para aprofundar as relações aqui evidenciadas e já previstas pela literatura especializada. Um importante ponto de investigação está justamente na identificação do ponto de transição entre CI e CC, ou seja, quando a motivação para a compra deixa de ser uma resposta a estímulos externos e torna-se patológica.

0 modelo proposto não tem elementos que viabilizem a avaliação dessa transição, uma vez que negligencia fatores psicológicos antecedentes do processo de CC. Está claro que o comprador compulsivo é incapaz de controlar seu impulso, isso, inclusive, decorre da própria definição de CC, entretanto, parece ser razoável, 
conceitualmente, que para haver a transição entre CI e CC é necessária a existência de outros antecedentes como ansiedade, instabilidade psicológica ou até mesmo desiquilíbrios de natureza hormonais.

Além disso, seria adequada a replicação desta pesquisa com o suporte de outra escala para medir os traços de personalidade, a fim de descobrir se a mudança do instrumento trará resultados distintos destes encontrados, bem como a comparação de eficiência dos instrumentos com amplitudes diferentes de mensuração $(5,7,11$ pontos de Likert), com o propósito de ratificar a diferença aqui encontrada em relação a esta variabilidade. Para pesquisas futuras, sugere-se a utilização da variável mediadora gênero, que por uma questão de foco, não foi abordada neste estudo e a inclusão de outros antecedentes, como ansiedade, para explicar a CC.

Finalmente, deve-se ressaltar que os resultados apresentados não são passíveis de generalização devido à utilização de amostra não probabilística de estudantes universitários, o que se configura como uma fraqueza da pesquisa. Faz-se interessante, portanto, replicar este trabalho em segmentos distintos da população, que apresentam hábitos de consumo diferentes, com o intuito de se verificar se essas relações também podem ser constatadas.

\section{REFERÊNCIAS}

Almeida, A. R., \& Botelho, D. (2006). Construção de questionários. In Botelho, D., \& Zouain, D. M. Pesquisa quantitativa em administração. São Paulo: Atlas.

Araújo, G. P., Ramos, A. S. M. R. (2010). Comportamento de compra por impulso em shopping centers: pesquisa com consumidores de Brasília-DF e Natal-RN. Revista Eletrônica de Administração, 16(3), p. 589-610.

Aravena, V., Herrera, V., Poblete, P., \& Vera, D. (2006). Consumo patológico: Compra Impulsiva y Compulsiva. Universidad De La Frontera. Recuperado em 26 de dezembro, 2016,

de http://www.inpsicon.com/estudios_realizado s/espanol/Aravena_Esp_04052007.pdf

Baik, J., Lee, K., Lee, S., Kim, Y., \& Choi, J. (2016). Predicting personality traits related to consumer behavior using SNS analysis. New Review of Hypermedia and Multimedia, 22(3), p. 189-206.
Baker, A. M., Moschis, G. P., Rigdon, E. E, \& Fatt, C.K. (2016). Linking family structure to impulsecontrol and obsessive-compulsive buying. Journal of Consumer Behavior, 15(4), p. 291302.

Basso, K. (2008). Personalidade e lealdade: Uma aplicação do modelo meta-teorico de motivação e personalidade (modelo 3M). Dissertação de mestrado, Universidade Católica do Rio Grande do Sul, Porto Alegre.

Basso, K., Espartel, B.I., Sampaio, C. H., Perin, M. G., \& Antoni, V. L. (2009, janeiro/abril). Personalidade e lealdade: Proposições de pesquisa com o modelo metateórico de motivação e personalidade. Revista Economia \& Gestão, 19(19), 102-124.

Baumgartner, H. J. (2002). Toward a personology of the customer. Journal of Consumer Research, 29(2), p. 286-292.

Beatty, S., \& Ferrel, E. (1998). Impulse buying: modeling its precursors. Journal of Retailing, 74(2), 169-191.

Costa, F. C. (2002). Relacionamento entre influências ambientais e o comportamento de compra por impulso: Um estudo em lojas físicas e virtuais. Tese de doutorado, Universidade de São Paulo, São Paulo.

Davidoff, L. (1983). Introdução à psicologia. São Paulo: McGraw-Hill.

Dias, S. E. F. et al. (2014). Efeitos das estratégias de marketing de compras coletivas sobre o comportamento impulsivo. REMark, 13 (3), 138-151.

Donovan, R. J., Rossiter, J. R., Marcoolyn, G., \& Nesdale, A. (1994). Store atmosphere and purchasing behavior. Journal of retailing, 70(3), 283-294.

Faber, R., \& O'guinn, T. (1992). A Clinical Screener for Compulsive Buying. Journal of Consumer Research, 19(1), 459-469.

Ferreira, V. (2008). Psicologia Econômica: Estudo do comportamento econômico e da tomada de decisão. Rio de Janeiro: Elsevier.

Fontenelle, I. A. (2008). Psicologia e Marketing: da Parceria a Crítica Arquivos Brasilerios de Psicologia, 60(2), 143-57.

Goldberg, L. R. (1992). The development of markers for the Big-Five factor structure. Psychological Assessment, 4(1), 26-42.

Hair, J.F. et al. (2005). Análise multivariada de dados (5a ed.). Porto Alegre: Bookman.

Harris, E. G., \& Mowen, J. C. (2001). The influence of cardinal-, central-, and surface-level personality traits on consumers' bargaining and complaint intentions. Psychology \& Marketing, 18 (11), p. 1155-1185.

Hutz, C. S., Nunes, C. H., Silveira, A. D., Serra, J., Anton, M., \& Wieczorek, L. S. (1998). 0 
desenvolvimento de marcadores para a avaliação da personalidade no modelo dos cinco grandes fatores. Psicologia: Reflexão $e$ Crítica, 11(2), 395-411.

Kassarjian, H. H. (1971). Personality and Consumer Behavior: A Review. Journal of Marketing Research, 8(4), 409-418.

Kukar-Kinney, M., Scheinbaum, A. C., \& Schaefers, T. (2016). Compulsive buying in online daily deal settings: An investigation of motivations and contextual elements. Journal of Business Research, 69(2), 691-699.

Lucas, M., \& Koff, E. (2014). The role of impulsivity and of self-perceived attractiveness in impulse buying in women. Personality and Individual Differences, 56(1), 111-115.

Matos, C., \& Bonfanti, K. (2008). Comportamento Compulsivo de Compra: Fatores Influenciadores no Público Jovem. Anais do XXXII ENANPAD. Rio de Janeiro, RJ.

Monteiro, P. R. (2006). Personalidade, compra compulsiva, hábitos e inovação em moda: Uma aplicação do modelo 3M de motivação e personalidade. Dissertação de mestrado, Universidade Federal de Minas Gerais, Minas Gerais.

Monteiro, P. R. R., Veiga, R. T., \& Gonçalves, C. A. (2009, julho/dezembro). Previsão de comportamentos de consumo usando a personalidade. Revista de Administração de Empresas Eletrônica, São Paulo, 8(2).

Mowen, J. C. (2000). The $3 \mathrm{M}$ model of motivation and personality: Theory and Empirical Applications to Consumer Behavior. Boston: Kluer Academic Publishers.

Mowen, J., \& Spears, N. (1999). Understanding compulsive buying among college students: A hierarchical approach. Journal of Consumer Psychology, 8(1), 407-425.

O'guinn, T. C., \& Faber, R. J. (1989, setembro). Compulsive Buying: A Phenomenological Exploration. Journal of Consumer Research, 16, 147-157.

Otero-López, J. M., \& Villardefrancos, E. (2013). Fivefactor model personality traits, materialism, and excessive buying: A mediational analysis. Personality and Individual Differences, 54(6), 767-772.

Pervin, L. A., \& John, O. P. (2004). Personalidade: teoria e pesquisa. Porto Alegre: Artmed.

Piron, F. (1991). Defining impulse purchasing. Advances in Consumer Research, 18, 509-514.

Porpino, G., \& Parente, J. (2013). Antecedentes e consequências da compra impulsiva: Um Estudo Exploratório. Congresso online Gestão, educação e promoção da saúde, 2. São Paulo.
Roberts, J., \& Manolis, C. (2012). Cooking up a recipe for self-control: The three ingredients of selfcontrol and its impact on impulse buying. Journal of Marketing Theory \& Practice, 20, 173-188.

Roberts, J. A., Manolis, C., \& Pullig, C. (2014). Contingent self-esteem, self-presentational concerns, and compulsive buying. Psychology \& Marketing, 31(2), 147-160.

Rook, D. (1987). The buying impulse. Journal of Consumer Research, 14, 189-199.

Rook, D., \& Fisher, R. (1995, dezembro). Normative influences on impulsive buying behavior. Journal of Consumer Research, 22(3), 305-313.

Rook, D., \& Hoch, S. (1985). Consuming impulses. Advances in Consumer Research, 12, 23-27.

Saucier, G. (1994). Mini-Markers: a brief version of Goldberg's unipolar big-five markers. Journal of Personality Assessment, 63, 506-516.

Santos, A. A. A., Sisto, F. F., \& Martins, R. M. M. (2003, janeiro/junho). Estilos cognitivos e personalidade: um estudo exploratório de evidências de validade. Psico-USF, 8(1), 11-19.

Serviço de Proteção ao Crédito. 52\% dos brasileiros fizeram alguma compra por impulso nos últimos três meses, revela SPC Brasil. Recuperado em 20 de fevereiro, 2016, de https://www.spcbrasil.org.br/pesquisas/pes quisa/905.

Shahjehan, A., Qureshi, J. A., Zeb, F., \& Saifullah, K. (2012). The effect of personality on impulsive and compulsive buying behaviors. African Journal of Business Management, 6(6), 2187.

Shehzadi, K., Ahmad-ur-Rehman, M., Cheema, A. M., \& Ahkam, A. (2016). Impact of Personality Traits on Compulsive Buying Behavior: Mediating Role of Impulsive Buying. Journal of Service Science and Management, 9(5), 416-432.

Silva, R. S., Schlottfeldt, C. G., Rozenberg, M. P., Santos, M. T., \& Lelé, A. J. (2007). Replicabilidade do Modelo dos Cinco Grandes Fatores em medidas da personalidade. Mosaico: Estudos em Psicologia, 1(1), 37-40.

Sun, T., Wu, G., \& Youn, S. (2004). Psychological antecedents of impulsive \& compulsive buying: A hierarchical perspective. The Proceedings of the Society for Consumer Psychology 2004 Winter Conference, 168-174.

Stock, R. M., Hippel, E. V., \& Gillert, N. L. (2016) Impacts of personality traits on consumer innovation success. Research Policy, 45, 757769.

Tinne, W. (2011). Factors affecting impulse buying behavior of consumers at superstores in Bangladesh. ASA University Review, 5, 209220.

Valence, G., D'astous, A., \& Fortier, L. (2004). Compulsive buying: Concept and 
measurement. Journal of Comsumer Policy, 11(4), 419-433.

Vasconcellos, S. J. L., \& Hutz, C. S. (2008). Construção e validação de uma escala de abertura à experiência. Revista Avaliação Psicológica, 7, $135-141$.

Veludo, O. T., Ikeda, A, \& Santos, R. (2004). Compra compulsiva e cartão de crédito entre universitários. Revista de Administração de Empresas, São Paulo, 44(3), 89-99.

Verplanken, B., \& Herabadi, A. (2001). Individual differences in impulse buying tendency: Feeling and no thinking. European Journal of personality, 15(1), 71-83.
Youn, S. (2000). The dimensional structure of consumer buying impulsivity: Measurement and validation. Tese de doutorado, University of Minnessota, Minnesota.

Youn, S., \& Faber, R. (2000). Impulse buying: its relations to personality traits and cues. Advances in Consumer Research, 27, 179-186.

Zanella, V. (2001). How to curb impulse buying tendencies. Recuperado em 26 de dezembro, 2016, de http://utut.essortment.com/impulsebuying_r htb.htm.

Goldberg, L. R. (1992, Março). The development of markers for the Big-Five factor structure. Psychological Assessment, 4(1), 26-42.

\title{
Analysis of the Relations Among Personality Traits, Impulsive Buying Behavior and Compulsive Buying Disorder
}

\begin{abstract}
This study aims to identify the relations among personality traits, impulsive buying behavior and compulsive buying disorder in comparison to the structural model proposed by Sun, Wu and Youn (2002). A quantitative survey was conducted through a sample of 424 participants, randomly divided into two groups of 212 participants. Two confirmatory factorial analyzes were performed on different samples and the structural model was tested using SPSS and AMOS software. The results indicate that neuroticism and extroversion are positively related to $I C$, as well as a positive association between impulsive buying behavior and compulsive buying disorder. The paper presents a methodological contribution on the operationalization of the scales and brings a critique to the subspecific nature of the relationship between impulsive and compulsive buying.

Keywords: Personality traits; Impulsive buying behavior; Compulsive buying disorder.
\end{abstract}

